\title{
Microwave phased array for aerological radar
}

\author{
Sergey Shabunin ${ }^{1,}$, Sergey Plokhov ${ }^{1}$, Ilia Bukrin $^{1}$, and Victor Chechetkin ${ }^{1}$ \\ ${ }^{1}$ Ural Federal University, 620002, Ekaterinburg, Russia
}

\begin{abstract}
The use of radars with phased antenna arrays in aerological atmospheric sounding systems significantly increases the technical characteristics of the radio channel, ensures reliable auto-tracking in the near zone at high angular velocities of the aerological probe, and reduces the overall dimensions of the radar station. Modelling and optimization of the parameters of the phased array and phase shifters were carried out in the NI AWR Design Environment. The results of the study showed that when designing a radar station, it is possible to reduce the level of side lobes of the phased array antenna pattern and the effect of reflections from the underlying surface under operating conditions significantly.
\end{abstract}

\section{Introduction}

Radio sounding systems (RSS) of the Earth atmosphere obtain the most accurate aerological information about the thermodynamic parameters of the atmosphere, such as temperature, humidity, pressure, wind direction and speed at altitudes from the ground level up to $35-40 \mathrm{~km}$. Two kinds of RSS, such as radar and radio navigation systems, operate now in the Russian Federation [1, 2]. The Roshydromet network uses Vector-M and MARL radars for meteorological monitoring.

A common problem of the radar RSS is the effect of an antenna beam reflection from the underlying surface of the ground in the near region at the beginning of the aerological radiosonde flight and in the far zone at a long distance from the observation point with small elevation angles.

We use the amplitude comparison method in angular coordinates for automatic radiosonde tracking. Therefore, lowering the side lobes level (SLL) of the antenna radiation pattern and accurate angular scanning is an important task for the modern radar design.

\section{Objective}

The authors proposed in [1] an antenna array with switched beams for contact monitoring of the atmosphere. Later a meteorological radar "Breeze" was developed based on this antenna array.

The antenna array consists of four subarrays, which meets the requirements of the control simplicity and reliability of the design.

Phase switching between the subarrays made it possible to provide a beam scan with a level of overlap of the lobes at the level of minus 1.2-2.0 dB. During operation, a deficiency

* Corresponding author: s.n.shabunin@urfu.ru 
of the antenna system was revealed in the form of a sufficiently high sidelobe level in the direction of the Earth's surface, leading to the capture of a false target reflected from the Earth. The objective of this study was to develop a novel antenna system that would have a significantly lower SLL while maintaining ease of control and reliability of the radar design.

\section{Radiation characteristics}

The radar has a wide beam mode because of the high angular velocity of the radiosonde at the initial stage of the flight and the need to quickly capture and track the target. This mode is implemented using a four-element patch antenna array, located in the center of the main antenna array (Fig. 1). Four subarrays, consisting of 18 patch antennas each, realize the narrow beam mode. The problem of the optimal placement of the subarrays and a fourelement array has been solved. Approximate calculations of the radiation pattern of an array with seventy-six elements were made using the MATLAB. For this antenna, we chose square printed patches connected with microstrip lines and grounding in the center, where the potential is equal to zero. The step between the elements is the wavelength because there is no dielectric substrate used in this design. The condition for optimal target resolution is radiation overlapping during scanning of minus 1.5-2.0 dB. Fig. 2 shows the factor of the phased antenna array when all the elements are phased separately (ideal case).

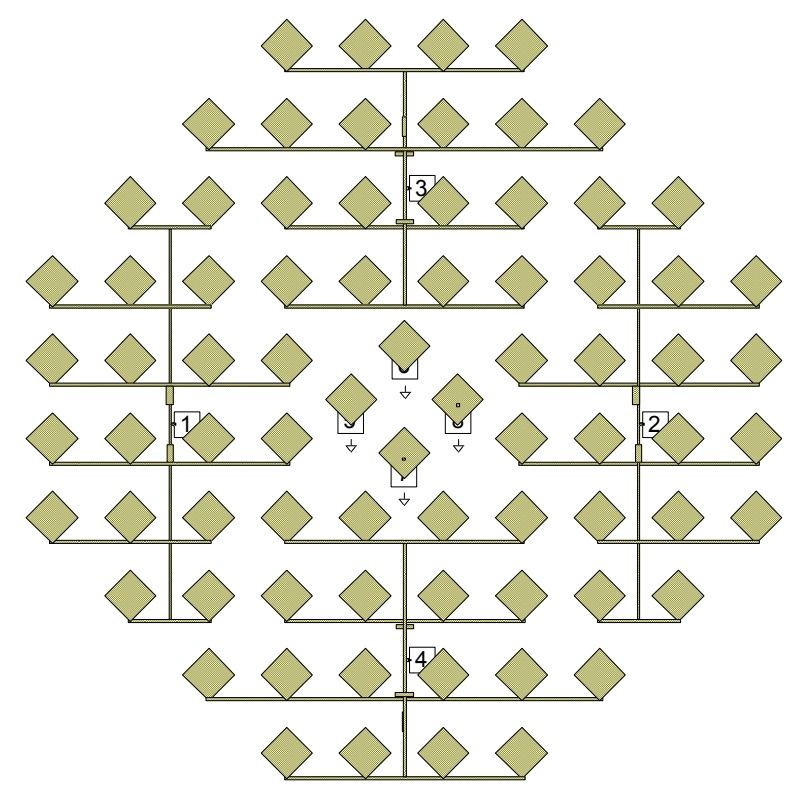

Fig. 1. The layout of the proposed antenna array.

The radiation pattern maximum deflection is 2.5 degrees. The SLL is $-17.5 \mathrm{~dB}$. Diffraction maxima decrease to an acceptable level due to the directional properties of the printed radiating element.

The main disadvantage of elementwise phasing is the need to have a large number of phase shifters. Besides, phase shifters must provide an arbitrary phase shift from 0 to 360 degrees with a given discrete. In (1), the phasing of four subarrays was applied. This design is reliable, allowing you to have simple control devices and phase shifters with a fixed phase shift. The disadvantage of this solution is the long distance between the phase centers 
of the subarrays. It causes the appearance of diffraction maxima and an increase in the SLL for the regular structure of the antenna.
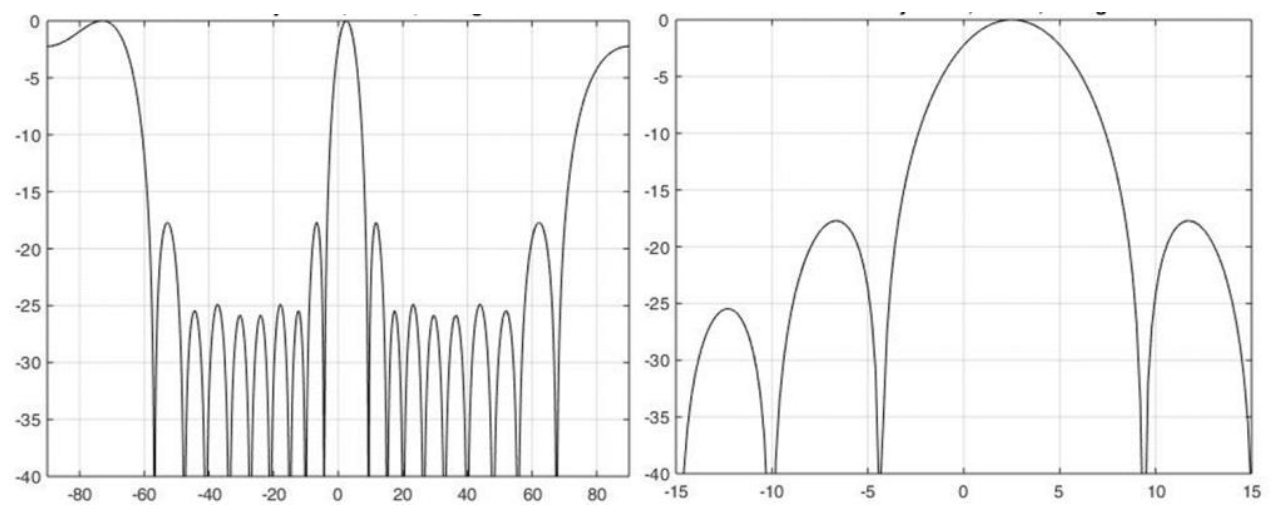

Fig. 2. 76-element antenna array factor.

In the proposed design of the antenna array, we provide an acceptable SLL due to the intersection in the phasing planes of the subarrays excited with different phase shifts. Preliminary analysis showed that the selected layout for the antenna design allows you to reduce the SLL by 5-7 dB. Fig. 3 shows the antenna array factor for a phased antenna array, consisting of four 18-element subarrays and a central 4-element small phased antenna array. The phase shift of 60 degrees between the subarrays provides the required level of overlapping of the maxima of radiation patterns at the level of minus $2 \mathrm{~dB}$.
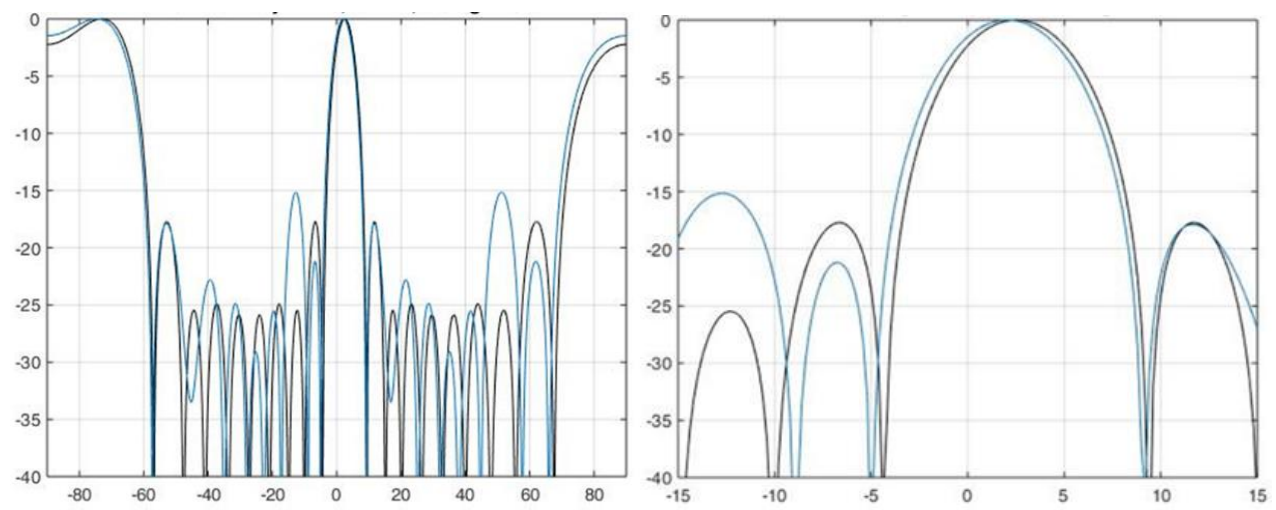

Fig. 3. Comparison of the antenna array factors for elementwise phasing (black line) and phasing of subarrays (blue line)

We created the antenna model in the NI AWR Design Environment. The main goal in the proposed design was to reduce the SLL in the elevation plane (E-plane).

Fig. 4 and 5 show the radiation patterns of a 4-element antenna array mounted in the center. The necessary level of overlap of radiation patterns is achieved at the same phase shifts as in the main antenna. For a beam deflected downward relative to the normal to the plane of the antenna, the gain is $15.5 \mathrm{~dB}$, the width of the main lobe at the half-power level is 27.8 degrees, and the decay of radiation along the normal is $2.1 \mathrm{~dB}$. For a beam rejected upward, the gain is $15.3 \mathrm{~dB}$, the width of the main lobe at the half-power level is 27.3 degrees, and the decay of radiation along the normal is $1.9 \mathrm{~dB}$. The deviation of the beam in the azimuthal plane to the left and right relative to the normal is symmetric, which follows from the symmetry of the array design in the H-plane (Fig. 5). The gain of the small phased array is $15.5 \mathrm{~dB}$, the width of the main lobe at the half-power level is 27.9 degrees, SLL is 
minus14.3 dB, and the level of overlapping radiation patterns in the normal direction is minus $2.4 \mathrm{~dB}$.

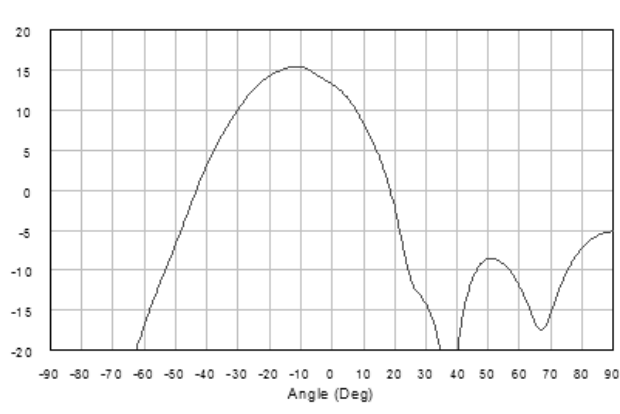

(a)

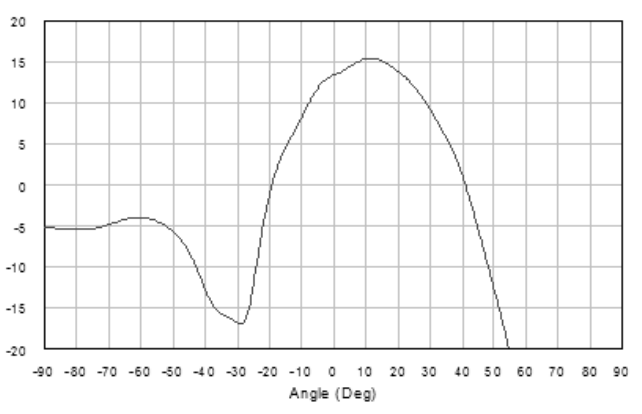

(b)

Fig. 4. Radiation pattern of small array when the beam is deflected down (a) and up (b) in the elevation plane

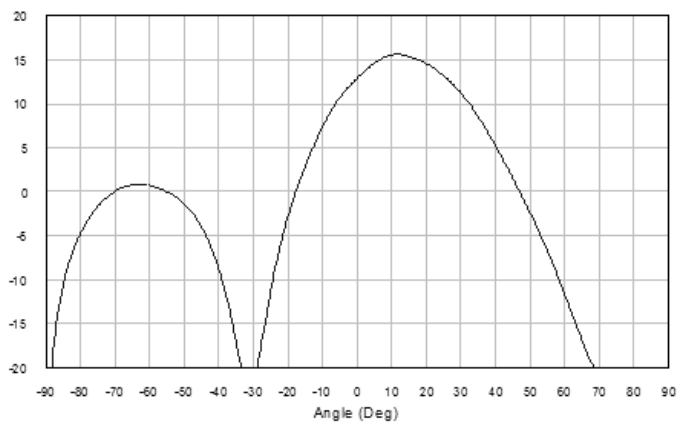

Fig. 5. Radiation pattern of small array when the beam is deflected in the azimuthal plane.

Fig. 6 and 7 show the radiation patterns of the phased antenna array in the narrow beam mode. For a beam deflected downward relative to the normal to the plane of the antenna, the gain is $27.4 \mathrm{~dB}$, the width of the main lobe at a half-power level is 5.9 degrees, and the decay of radiation along the normal is $1.7 \mathrm{~dB}$. For the beam tilted up, the gain is $27.4 \mathrm{~dB}$, the width of the main lobe at the half-power level is 5.9 degrees, and the decay of the radiation along the normal is $2.1 \mathrm{~dB}$. The deviation of the beam in the azimuthal plane to the left and right relative to the normal is symmetric, which follows from the symmetry of the antenna design in the H-plane (Fig. 7). The gain of the phased antenna array is $27.7 \mathrm{~dB}$, the width of the main lobe at the half-power level is 5.9 degrees, SLL is minus $14.9 \mathrm{~dB}$, and the level of overlapping radiation patterns in the normal direction is minus $1.9 \mathrm{~dB}$.

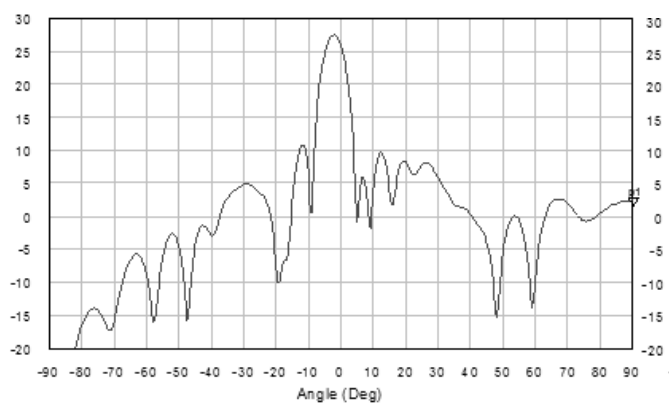

(a)

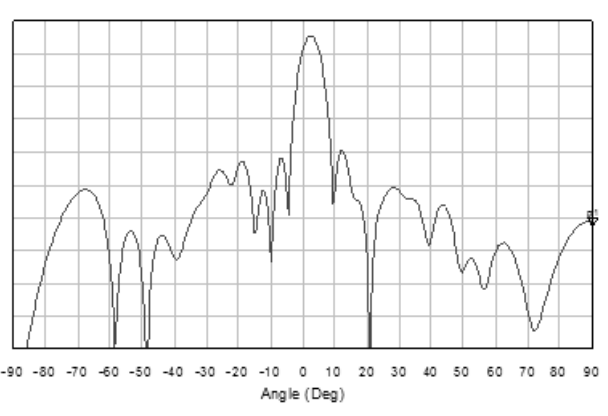

(b)

Fig. 6. Radiation patterns of the phased antenna array when the beam is deflected down (a) and up (b) in the elevation plane. 


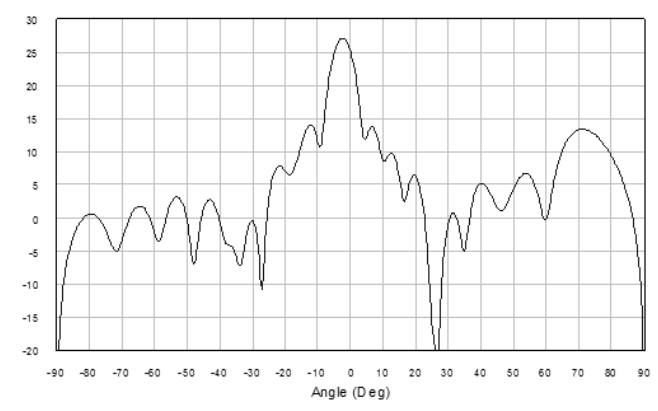

Fig. 7. Radiation pattern for beam deflection in the azimuthal plane.

\section{Antenna design}

The following design was developed to implement the desired parameters:

- the base of the antenna is an octagon of duralumin with dimensions of $1810 \times 1810 \mathrm{~mm}$ and a side height of $50 \mathrm{~mm}$ (Fig. 8);

- patch antennas are installed on the base through the aluminum bushings;

- the base is closed with the cover of expanded polystyrene to protect the elements from precipitation;

- there is an instrument compartment on the rear side of the antenna in which there is a transceiver module with a power amplifier, phase shifter and LNA (Fig. 9).

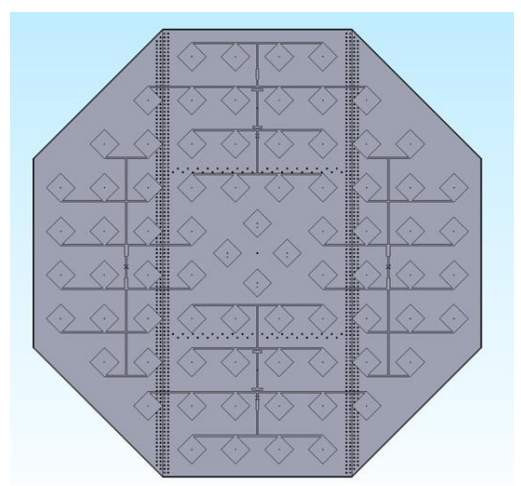

Fig. 8. The appearance of the antenna (top view).

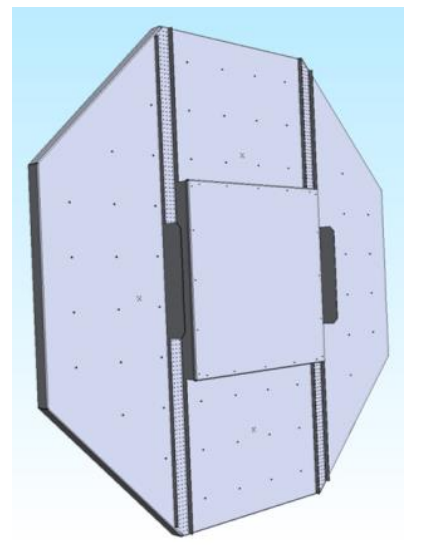

Fig. 9. The appearance of the antenna (bottom view). 


\section{Conclusion}

A phased antenna array has been developed that provides high technical characteristics of the meteorological radar, with the simplicity of design and control of a beam scanning. A four-element small phased array implements wide beam mode at short ranges. Due to the selected layout with overlapping subarrays, the SLL is reduced by 5-7 dB against the conventional design with switched radiation patterns. The simulation was performed using the MATLAB at the initial stage of the design and the NI AWR Design Environment.

Authors are grateful for the simulation software NI AWR Design Environment.

\section{References}

1. V.N. Kiselev, A.D. Kuznetzov, Sounding methods of the environment (of the atmosphere) (St. Petersburg Pablishers, 2004)

2. V.E. Ivanov, A.V. Gusev, K.A. Ignatkov, S.I. Kudinov, I.V. Malyigin, V.Ya. Noskov, O.V. Plohih, V.V. Ryisev, O.A. Chernyih, Uspekhi sovremennoi radioelektroniki, 9, 3 (2015)

3. D.I. Voskresenkii, etc., On-board antenna arrays and their elements (Radiotechnics, 2013)

4. D.I. Voskresenkii, etc., Active phased antenna arrays (Radiotechnics, 2004)

5. D.I. Voskresenkii, etc., Antennas and microwave devices. Phased Array Design (Radio and communications, 1994) 